\title{
Age influences structural brain restoration during weight gain therapy in anorexia nervosa
}

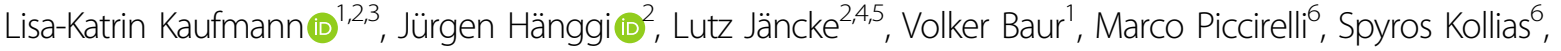 \\ Ulrich Schnyder ${ }^{7}$, Chantal Martin-Soelch ${ }^{3}$ and Gabriella Milos ${ }^{1}$
}

\begin{abstract}
Neuroimaging studies on anorexia nervosa (AN) have consistently reported globally reduced gray matter in patients with acute AN. While first studies on adolescent AN patients provide evidence for the reversibility of these impairments after weight gain, longitudinal studies with detailed regional analysis for adult AN patients are lacking and factors associated with brain restitution are poorly understood. We investigated structural changes in anorexia nervosa using T1-weighted magnetic resonance images with surface-based morphometry. The sample consisted of 26 adult women with severe $\mathrm{AN}$ and 30 healthy controls. The longitudinal design comprised three time points, capturing the course of weight-restoration therapy in AN patients at distinct stages of weight gain (BMI $\leq 15.5 \mathrm{~kg} / \mathrm{m}^{2} ; 15.5<\mathrm{BMl}$ $<17.5 \mathrm{~kg} / \mathrm{m}^{2} ; \mathrm{BMI} \geq 17.5 \mathrm{~kg} / \mathrm{m}^{2}$ ). Compared to controls, AN patients showed globally decreased cortical thickness and subcortical volumes at baseline. Linear mixed effect models revealed the reversibility of these alterations, with brain restoration being most pronounced during the first half of treatment. The restoration of cortical thickness of AN patients negatively correlated with age, but not duration of illness. After weight restoration, residual group differences of cortical thickness remained in the superior frontal cortex. These findings indicate that structural brain alterations of adult patients with severe AN recuperate independently of the duration of illness during weight-restoration therapy. The temporal pattern of brain restoration suggests a decrease in restoration rate over the course of treatment, with patients' age as a strong predictor of brain restitution, possibly reflecting decreases of brain plasticity as patients grow older.
\end{abstract}

\section{Introduction}

Anorexia nervosa (AN) is a severe and enduring psychiatric disorder, characterized by significantly reduced body weight ${ }^{1}$ and associated with reduced brain matter ${ }^{2,3}$. In order to better understand the hitherto unclear psychopathology of the illness, these brain alterations are being examined more closely using magnetic resonance imaging (MRI).

Recent studies on adult AN patients have largely established reduced gray matter volume in a globally

\footnotetext{
Correspondence: Lisa-Katrin Kaufmann (lisa-katrin.kaufmann@usz.ch) ${ }^{1}$ Department of Consultation-Liaison Psychiatry and Psychosomatics, University Hospital Zurich, University of Zurich, Zurich, Switzerland

${ }^{2}$ Division of Neuropsychology, Department of Psychology, University of Zurich, Zurich, Switzerland
}

Full list of author information is available at the end of the article distributed set of brain regions, affecting almost the whole corte $^{3-5}$. Reports on reduced white matter volumes have been less consistent, with some studies reporting decreased volume ${ }^{6,7}$, while others did not find significant changes $^{8,9}$. These reductions are generally interpreted as consequences of patients' malnutrition and are mostly absent in long-term recovered AN patients ${ }^{10-15}$. However, reports on persevering gray matter decreases in regions such as the precuneus ${ }^{11}$, the anterior cingulate cortex, and the supplementary motor area ${ }^{16}$, as well as persisting volume increases in the insula, the orbitofrontal cortex ${ }^{17,18}$, and the post-central gyrus ${ }^{19}$ paint a more complex picture. Longitudinal study designs are thus required to address the question of brain regeneration in women with AN. In adolescent AN patients and a mixed-sample of adolescents and young adults, three longitudinal

\section{(c) The Author(s) 2020}

(c) Open Access This article is licensed under a Creative Commons Attribution 4.0 International License, which permits use, sharing, adaptation, distribution and reproduction in any medium or format, as long as you give appropriate credit to the original author(s) and the source, provide a link to the Creative Commons license, and indicate if changes were made. The images or other third party material in this article are included in the article's Creative Commons license, unless indicated otherwise in a credit line to the material. If material is not included in the article's Creative Commons license and your intended use is not permitted by statutory regulation or exceeds the permitted use, you will need to obtain permission directly from the copyright holder. To view a copy of this license, visit http://creativecommons.org/licenses/by/4.0/. 
studies report complete normalization of gray matter loss after a brief period of weight restoration ${ }^{20-22}$. For adult AN patients, only global tissue volumes have been investigated $^{23,24}$, but longitudinal studies with detailed regional analyses are lacking.

In this longitudinal study, we aimed to identify and monitor disease-specific structural brain alterations in patients with severe AN using three time points: (TP1) at the beginning of inpatient treatment in a stage of severe underweight, (TP2) during inpatient treatment after initial weight gain, and (TP3) at discharge with a body mass index $\geq 17.5\left(\mathrm{BMI} ; \mathrm{kg} / \mathrm{m}^{2}\right)$. Based on previous results ${ }^{3,4}$, we expected decreases in cortical and subcortical gray matter of AN patients in the acute stage compared to healthy controls (HC). Over the course of weightrestoration treatment, we expected a regeneration of these gray matter compared to HC. Additionally, we explored whether restoration of brain structure in AN patients was associated with a shorter duration of illness, with improvements in BMI, eating disorder-related cognitions, or with a younger age at the time of treatment.

\section{Materials and methods Participants}

We recruited 26 women diagnosed with severe AN (22 restrictive, 4 binge/purge; $18-32$ years) and $30 \mathrm{HC}$ (BMI $18.5-23.0 \mathrm{~kg} / \mathrm{m}^{2} ; 18-30$ years). Inclusion criteria for the AN group comprised severe underweight (BMI $\leq 15.5 \mathrm{~kg} /$ $\mathrm{m}^{2}$ ) to allow for a homogeneous weight development and to capture brain changes at distinct phases of weight gain (see "Study design"). Both groups were assessed with the Structured Clinical Interview for DSM-IV-TR Axis I Disorders ${ }^{25}$ and matched for sex, age, handedness, intelligence and years of education. All women were strongly right-handed, except for one left-handed woman in each group $^{26}$. Fifteen patients were given comorbid diagnoses: major depression (12), major depression and social anxiety disorder (2), and obsessive-compulsive disorder (1). Twelve patients were receiving medication when they entered the study; these patients were instructed to continue taking them as prescribed (see Table S1 for further details). Exclusion criteria comprised current or past neurological disorders, substance abuse or addiction, contraindications to MRI, and for $\mathrm{HC}$ a history of eating disorders or any mental illness, first- or second-degree relatives with a lifetime diagnosis of an eating disorder, and the use of any medication, including hormonal contraceptives.

Data of two patients were not available at the first time point due to technical difficulties. This sample partially overlaps with the sample of a recent publication of our group, focusing on white matter alterations in the for$\operatorname{nix}^{27}$. The study was approved by the local ethics committee and the study protocol complied with the
Declaration of Helsinki. All participants gave their written informed consent prior to study enrollment.

\section{Study design}

Patients with AN participated in an eating disorderspecific inpatient therapy program with a target $\mathrm{BMI} \geq$ $18.5 \mathrm{~kg} / \mathrm{m}^{2}$. Measurements of the AN group were taken at three time points: at the beginning (TP1; BMI $\leq 15.5 \mathrm{~kg} /$ $\mathrm{m}^{2}$ ), in the middle (TP2: $15.5<\mathrm{BMI}<17.5 \mathrm{~kg} / \mathrm{m}^{2}$ ), and towards the end of treatment with a BMI of $\geq 17.5 \mathrm{~kg} / \mathrm{m}^{2}$ (TP3; see Supplement for further details). The HC group participated in the same procedure at two time points (TP1 and TP3). The time interval between TP1 and TP3 for both groups was 4-6 months after TP1 (see also Supplement). The patients' TP1 was scheduled after a medical stabilization period of at least 2 weeks with a fixed meal plan and extensive somatic checks, to exclude biases of under- or hyperhydration due to the condition of acute starvation $^{28,29}$.

\section{Behavioral and psychometric assessment}

Validated German versions of the following psychometric tests were used in this study: The Viennese Matrices Test (WMT) for non-verbal intelligence ${ }^{30}$ and the Vocabulary Test (WST) for verbal intelligence ${ }^{31}$, both presented as computer-based versions ${ }^{32}$. Eating disorder cognitions were assessed with the Eating Disorder Examination Questionnaire (EDE-Q) ${ }^{33}$, depression severity was captured with the Beck Depression Inventory $(\mathrm{BDI})^{34}$.

\section{MRI data acquisition}

Brain images were acquired with a 3.0 Tesla whole-body MRI system (Ingenia, Philips, Best, The Netherlands), equipped with a 32-channel head coil. Whole-brain 3D T1-weighted structural images were acquired using a 3D Turbo-Field-Echo sequence (for details, see Supplement). All images were checked for relevant clinical pathology or anomalies by a trained neuroradiologist.

\section{MRI data preprocessing}

Structural T1-weighted images were preprocessed with the FreeSurfer software suite version 6.0.0 (http://surfer. nmr.mgh.harvard.edu/). This automated analysis procedure has been widely used and its preprocessing steps have been described in detail elsewhere ${ }^{35}$. In brief, T1weighted images in stereotactic space are segmented into different tissue types, considering a priori anatomical information and each voxel's intensity value. To estimate cortical thickness and subcortical volumes, images were preprocessed with FreeSurfer's longitudinal stream ${ }^{36}$. For each subject an unbiased median template image was created $^{37}$ using robust, inverse consistent registration between each time point of a subject ${ }^{38}$. Data for each 
subject were then resampled to the respective template and preprocessed by (1) registration to Talairach space, (2) construction of brain mask and skull stripping, (3) normalization and registration to the probabilistic atlas $^{39,40}$, (4) segmentation of subcortical regions, (5) creation of spherical surface maps, (6) registration to cortical atlas, and (7) parcellation, initialized with common information from the within-subject template to improve statistical power and reliability ${ }^{36}$. For each time point, cortical thickness maps for both hemispheres were then extracted and smoothed with a 15-mm full-width at half-maximum Gaussian kernel. Additionally, global brain measures and subcortical structures (averaged over both hemispheres) provided by the FreeSurfer stream were investigated. To assure data quality, all preprocessed images were visually inspected and checked with FreeSurfer's quality assessment tool.

\section{MRI data analysis}

The longitudinal analyses of the cortical thickness were performed using linear mixed effects (LME) models, more specifically with the spatiotemporal extension for massunivariate data as distributed with FreeSurfer ${ }^{41,42}$. LME models offer a powerful and versatile approach for the analysis of longitudinal data, with the advantage of differentiating between-subject and within-subject sources of variance and handling unequal numbers of time points ${ }^{41}$. Visual inspection of mean trajectories revealed a linear trend over time; thus, the spatiotemporal model was fitted with the intercept as random effect. For each tested contrast (main effect group, main effect time, interaction effect group $\times$ time), significance maps were created, and false discovery rate corrected within FreeSurfer (across both hemispheres) at a significance level of $p<0.05$, using an adaptive linear two-stage procedure ${ }^{43}$ to control for multiple comparisons. Time was measured in days from TP1. The longitudinal analyses for subcortical volumes were conducted using univariate LME models implemented in FreeSurfer, with intracranial volume as covariate and the intercept as random effect. To assess the magnitude and direction of differences, these comparisons were followed up by paired $t$-tests within groups. Reported $p$-values are adjusted for multiple comparisons using the Holm-Bonferroni procedure ${ }^{44}$.

Cross-sectional comparisons of cortical thickness between groups at TP1 and TP3 were calculated using vertex-wise general linear models with group as independent and cortical thickness as dependent variable within FreeSurfer, using Monte-Carlo simulation (5000 permutations) to correct for multiple comparisons. To perform cross-sectional comparisons of volumetric measures, values for global cortical and subcortical volumes were extracted from FreeSurfer. Subcortical volumes were corrected for intracranial volume ((volume/intracranial volume) $\times 1000$ ). To check for normality of the data distribution, the Shapiro Wilk test was used. Group comparisons were performed using $t$ tests for independent samples, adjusted for multiple comparisons (separately for regional and global measures) using the Holm-Bonferroni procedure $^{44}$. All $t$-tests were performed using $\mathrm{R}$, version 3.5.0 ${ }^{45}$.

\section{Additional statistical analyses}

Within- and between-group comparisons of demographic and psychometric measures were performed using two-tailed $t$-tests for paired or independent samples, respectively. To check for normality of the data distribution, the Shapiro-Wilk test was used. Standardized mean differences are reported as Hedges' $g$, with the pooled standard deviation as standardizer ${ }^{46}$ (see Supplement for details). Associations between changes in gray matter (cortical thickness per hemisphere, cortical thickness within the clusters of reduced cortical thickness identified at TP1, subcortical volumes) and clinical parameters (BMI, BMI increase per week, duration of illness, eating disorder-related cognitions, and depression severity) as well as age (at the beginning of treatment) were calculated as Pearson correlations. These tests were performed using $\mathrm{R}$, version $3.5 .0^{45}$ and subsequently adjusted for multiple comparisons according to Holm-Bonferroni ${ }^{44}$.

\section{Results}

\section{Demographic and psychometric measures}

Group comparisons of demographic data at baseline (TP1) revealed no significant differences between AN patients and $\mathrm{HC}$ with respect to age, intelligence and education (Table 1). As expected, AN patients had significantly lower BMIs, higher eating disorder-related cognition (EDE-Q) and depression (BDI) scores than $\mathrm{HC}$ at TP1. Over the course of therapy, patients' BMI increased significantly compared to baseline (TP1 to TP2, $16 \%$ BMI increase; TP2 to TP3, 13\% BMI increase). Similarly, psychometric scores of the AN group improved and dropped below the clinical cut-offs at the end of treatment, but remained significantly elevated compared to HC (Table 1).

\section{Brain alterations during severe underweight (TP1)}

Global brain volumes at TP1 showed reduced cortical gray matter and heightened CSF in AN patients compared to $\mathrm{HC}$, and a trend for reduced global subcortical gray matter (Table 2). No differences were observed with respect to cerebral white matter. Furthermore, intracranial volumes did not differ between groups, ruling out overall smaller skulls in AN patients as explanation for the observed differences.

Vertex-wise group comparisons of cortical thickness at TP1 demonstrated that global volumetric gray matter 
Table 1 Group characteristics.

\begin{tabular}{|c|c|c|c|c|c|c|c|c|c|c|c|c|c|}
\hline & \multicolumn{10}{|c|}{ Group and time point } & \multirow{3}{*}{$\begin{array}{l}\text { AN1 vs. HC1 } \\
p\end{array}$} & \multirow{3}{*}{$\begin{array}{l}\text { AN3 vs. HC3 } \\
\text { p }\end{array}$} & \multirow{3}{*}{$\begin{array}{l}\text { AN1 vs. AN3 } \\
p\end{array}$} \\
\hline & \multicolumn{2}{|l|}{$\begin{array}{l}\text { AN1 } \\
(n=24)\end{array}$} & \multicolumn{2}{|c|}{$\begin{array}{l}\text { AN2 } \\
(n=26)\end{array}$} & \multicolumn{2}{|c|}{$\begin{array}{l}\text { AN3 } \\
(n=26)\end{array}$} & \multicolumn{2}{|c|}{$\begin{array}{l}\text { HC1 } \\
(n=30)\end{array}$} & \multicolumn{2}{|c|}{$\begin{array}{l}\text { HC3 } \\
(n=30)\end{array}$} & & & \\
\hline & $M$ & $S D$ & $M$ & $S D$ & $M$ & $S D$ & $M$ & $S D$ & $M$ & $S D$ & & & \\
\hline Age & 22.25 & 4.08 & - & - & - & - & 24.15 & 3.42 & - & - & 0.075 & - & - \\
\hline Age of illness onset & 16.70 & 3.08 & - & - & - & - & - & - & - & - & - & - & - \\
\hline Duration of illness & 5.59 & 4.30 & - & - & - & - & - & - & - & - & - & - & - \\
\hline Education & $13.30^{\mathrm{a}}$ & 2.53 & - & - & - & - & $14.69^{b}$ & 4.68 & - & - & 0.180 & - & - \\
\hline $\mathrm{BMI}\left(\mathrm{kg} / \mathrm{m}^{2}\right)$ & 14.25 & 1.08 & 16.55 & 0.68 & $18.41^{c}$ & 0.45 & 20.81 & 1.77 & 20.38 & 1.56 & $<0.001$ & $<0.001$ & $<0.001$ \\
\hline BMI increase per week & - & - & 0.24 & 0.07 & 0.10 & 0.04 & - & - & -0.01 & 0.04 & - & $<0.001$ & - \\
\hline Time after TP1 (weeks) & - & - & 9.16 & 4.16 & 22.04 & 16.26 & - & - & 25.91 & 8.66 & - & 0.283 & - \\
\hline EDE-Q total & 3.19 & 1.26 & 1.98 & 1.11 & $1.57^{\mathrm{a}}$ & 1.01 & $0.64^{b}$ & 0.58 & $0.55^{\mathrm{d}}$ & 0.57 & $<0.001$ & $<0.001$ & $<0.001$ \\
\hline EDE-Q eating concern & 3.16 & 1.33 & 1.84 & 1.15 & $1.41^{\mathrm{a}}$ & 1.11 & $0.43^{b}$ & 0.56 & $0.41^{d}$ & 0.56 & $<0.001$ & $<0.001$ & $<0.001$ \\
\hline EDE-Q restraint & 3.29 & 1.82 & 1.33 & 1.24 & $1.10^{\mathrm{a}}$ & 0.81 & $0.48^{b}$ & 0.60 & $0.28^{d}$ & 0.39 & $<0.001$ & $<0.001$ & $<0.001$ \\
\hline EDE-Q shape concern & 3.47 & 1.22 & 2.85 & 1.57 & $2.27^{\mathrm{a}}$ & 1.50 & $0.91^{b}$ & 0.88 & $0.84^{d}$ & 0.76 & $<0.001$ & $<0.001$ & $<0.001$ \\
\hline EDE-Q weight concern & 2.84 & 1.21 & 1.90 & 1.10 & $1.50^{\mathrm{a}}$ & 1.28 & $0.75^{b}$ & 0.75 & $0.67^{d}$ & 0.80 & $<0.001$ & 0.012 & $<0.001$ \\
\hline $\mathrm{BDI}$ & 22.83 & 9.93 & 17.77 & 9.40 & $10.74^{\mathrm{a}}$ & 7.93 & $3.48^{\mathrm{b}}$ & 3.82 & $2.37^{d}$ & 2.51 & $<0.001$ & $<0.001$ & $<0.001$ \\
\hline WMT & 121.13 & 17.64 & - & - & - & - & 127.83 & 15.81 & - & - & 0.153 & - & - \\
\hline WST & $105.26^{\mathrm{a}}$ & 8.24 & - & - & - & - & 105.70 & 10.43 & - & - & 0.865 & - & - \\
\hline
\end{tabular}

Age, age of illness onset, duration of illness, and education in years.

$B M I$ body mass index, $B D I$ Becks Depression Inventory total score (cut-off for clinical range $\leq 11^{66}$ ), EDE-Q Eating Disorder Examination Questionnaire score (cut-off for clinical range $\geq 2.09^{67}$ ), WMT Viennese Matrices Test, WST Multiple Choice Vocabulary Test.

${ }^{a}$ Data available only for $\mathrm{AN}=23$

${ }^{\mathrm{b}} \mathrm{HC}=29$ subjects

${ }^{\mathrm{C}} \mathrm{BMI} \geq 18.5: n=15$

${ }^{\mathrm{d}} \mathrm{HC}=27$ subjects.

differences reported above were likely driven by differences in cortical thickness. Compared to $\mathrm{HC}$, AN patients showed considerably decreased cortical thickness in clusters covering large parts of both hemispheres (Fig. 1a), sparing only parts of the medial prefrontal, sensorimotor, and insular cortex, as well as basal parts of the temporal lobe and the calcarine cortex. Separate analyses of the subcortical structures yielded reduced volumes in patients with AN compared to $\mathrm{HC}$ at TP1, with pronounced differences in the hippocampus and the thalamus (Table 2).

\section{Recovery processes with weight restoration}

LME analyses of cortical thickness yielded a significant interaction of group $\times$ time in each hemisphere, revealing cortical restoration in AN patients over the course of treatment (Fig. 1b). Within-group analysis demonstrated significant global restoration of cortical thickness in both hemispheres during the first (mean increase $=0.08 \mathrm{~mm}$ ) and second treatment phase (mean increase $=0.04 \mathrm{~mm}$ ), with a notably faster restoration during the first phase
(Fig. 2a). Despite the significant global restoration of cortical thickness (Fig. 1b), whole-brain comparisons at the end of treatment (TP3) yielded a significant cluster of residual cortical thinning in $\mathrm{AN}$ patients compared to $\mathrm{HC}$ in the right superior frontal cortex (Fig. 1c).

LME analyses of subcortical volumes yielded significant interactions of group and time for the following structures: amygdala, caudate nucleus, hippocampus, and thalamus ( $p s \leq 0.002$, corrected; Supplementary Table S2). Within-group comparisons of the subcortical structures demonstrated statistically significant volume increases in AN patients of the amygdala, caudate nucleus, hippocampus, and thalamus during the first phase of treatment. In the second phase of treatment, only volumes of the hippocampus and the thalamus further increased (Table 2). Volumes of the nucleus accumbens, pallidum, and putamen did not change significantly within the AN group, as well as in comparison with HC (Table 2, Supplementary Tables S2, S3). Group comparisons at TP3 yielded no longer significant differences in subcortical 


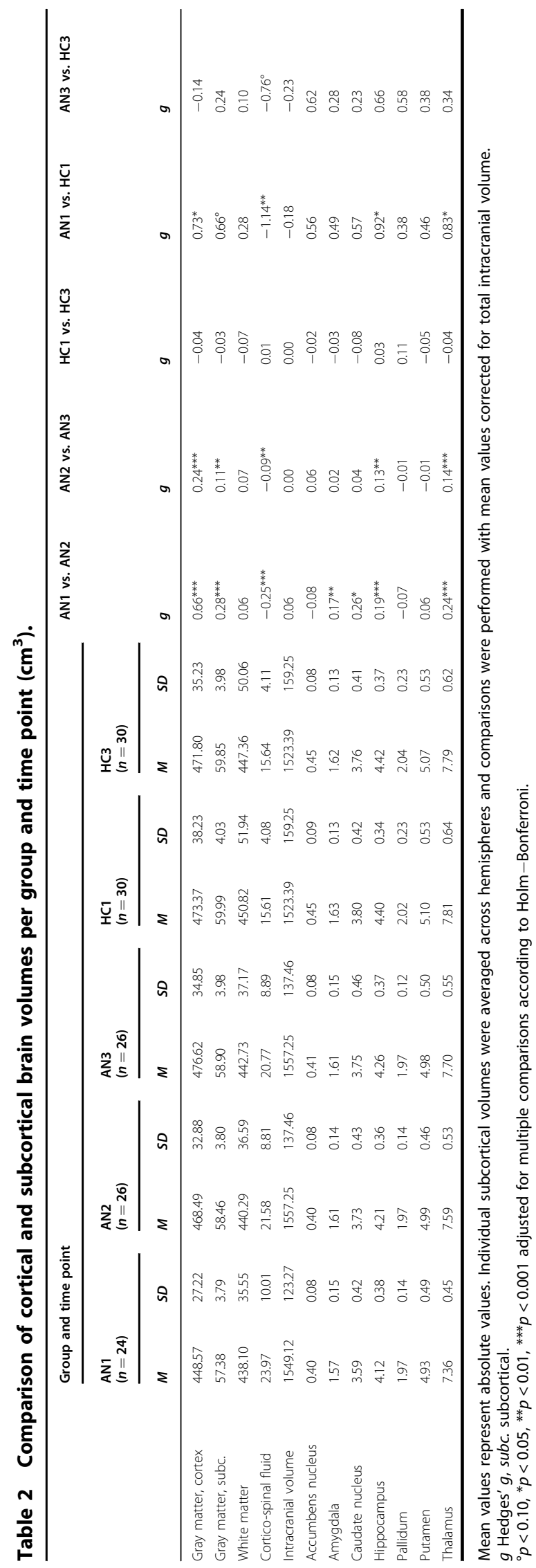

volumes, albeit slight remaining decreases (Table 2). There was no evidence that recovery processes were driven by AN subtype or patients' medication status (Supplementary Fig. S1, S2).

\section{Correlations with clinical and demographic parameters}

During the early phase of treatment (TP1-TP2), the recovery of subcortical structures was not associated with changes in clinical parameters, except for a positive association between the regeneration of the caudate nucleus and BMI increase $(r=0.68, p<0.01$, corrected; Supplementary Table S4). Similarly, BMI increase correlated with changes of cortical thickness of the right hemisphere on a trend level $(r=0.59, p=$ 0.06 , corrected; Table S5). Considering the homogeneity of BMI changes within the AN patient group, these associations underline the strong influence of body weight on structural brain measures.

During the later phase of treatment (TP2-TP3), the restoration of cortical thickness in both hemispheres was strongly correlated with patients' age, suggesting that patients' cortical thickness regenerated to a larger degree if patients were younger (Fig. 2b, c). Of note, this relationship persisted even after controlling for BMI at TP3 (left hemisphere, $r=-0.60, p=0.001$; right hemisphere, $r=-0.61, p<0.001)$ or the change of BMI between TP2 and TP3 (left hemisphere, $r=-0.60$, $p=0.001$; right hemisphere, $r=-0.60, p=0.001$ ). Furthermore, the reduction of cortical thickness at TP1 was independent of patients' age (Supplementary Fig. S3), indicating that younger patients' cortices were not thinner to start with. Other parameters were not associated with the increase of cortical thickness or subcortical volumes during this later phase of treatment.

\section{Discussion}

This longitudinal study investigated temporal patterns of cortical and subcortical regeneration in women with severe AN, assessing brain restoration at three distinct stages of weight gain. In the stage of severe underweight, our analyses revealed reduced cortical thickness as well as diminished subcortical volumes, which largely regenerated during a substantial weight gain up to a BMI of $\geq 17.5 \mathrm{~kg} / \mathrm{m}^{2}$. Specifically, patients' mean cortical thickness during the early phase of treatment increased markedly by $0.08 \mathrm{~mm}$ (TP1 to TP2, $16 \%$ BMI increase compared to baseline). During the later phase of treatment, further restitution of mean cortical thickness by $0.04 \mathrm{~mm}$ was observed (TP2 to TP3, 13\% BMI increase compared to baseline). However, significant residual differences remained in a right superior frontal cluster. Closer examination of associated factors revealed a strong influence of age and 


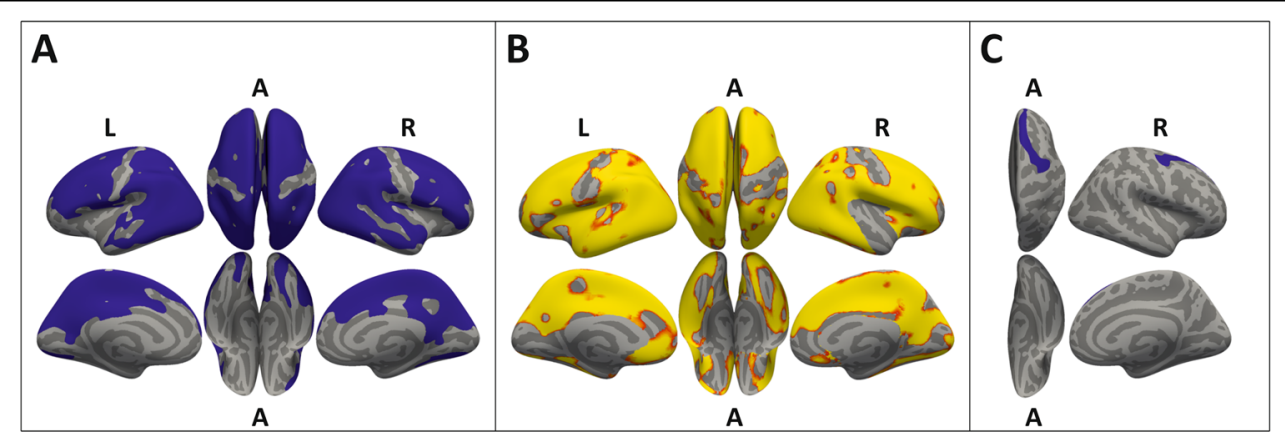

Fig. 1 Group comparisons of cortical thickness projected onto inflated brain surfaces (gray). a Reduced cortical thickness of AN patients compared to $\mathrm{HC}$ at the beginning of treatment (TP1). Significant clusters (blue, $p<0.05$ ), corrected for multiple comparisons using Monte-Carlo simulations (5000 permutations). b Increase of cortical thickness during weight restoration in AN patients. Significant interaction of group $\times$ time (red, $p<0.05$; yellow, $p<0.01$ ), controlled for multiple comparisons with FDR correction. $\mathbf{c}$ Residual cortical thinning in AN patients compared to HC at the end of treatment (TP3). A significant cluster (blue, $p<0.05$ ) remained in the superior frontal region (AN: mean $=2.71 \mathrm{~mm} \pm 0.15, \mathrm{HC}$ : mean $=$ $2.86 \mathrm{~mm} \pm 0.19$ ), after correcting for multiple comparisons using Monte-Carlo simulation (5000 permutations). A anterior, $L$ left, $R$ right.

BMI on cortical gray matter regeneration, suggesting the cortex of younger patients recovered to a larger extent during weight normalization. Apart from highlighting the impact of body weight on the structural measures of the brain, this may indicate a decrease of brain plasticity as patients grow older ${ }^{47}$.

The reversibility of decreases in cortical thickness and subcortical volumes is in overall agreement with findings of longitudinal studies in adolescent AN patients after a brief period of weight restoration ${ }^{20,21}$. In adolescents, BMI increases of at least $10 \%$ were accompanied by an average restitution of cortical thickness of $0.16 \mathrm{~mm}$ after a treatment duration of 12 weeks ${ }^{20}$. In comparison, the increases of cortical thickness in our sample added up to only $0.12 \mathrm{~mm}$ after a total treatment duration of 22 weeks. Recovery processes in adolescent AN patients might thus happen at a faster rate compared to adult patients. An alternative explanation for the differences in restoration rate might be the longer duration of illness and higher disease severity (indexed by the BMI) of patients in the current sample. However, this is not supported by the lack of correlation between illness duration and the regeneration of cortical thickness. Additionally, our finding of a persisting influence of age, after controlling for BMI and change in BMI, speaks against such an interpretation. An initially greater reduction of cortical thickness in younger patients may be another reason for the differing velocities of regeneration. However, we did not find evidence for an association between age and cortical thickness during the stage of acute starvation in the present sample. Lastly, the observed restoration rate might be influenced by the medical stabilization period before TP1 we used to mitigate inflated effects, which was not employed in the studies with adolescents. However, hydration status in adolescents was reported to be within the normal range ${ }^{20}$. Moreover, the residual cortical thinning at TP3 in our sample, compared to fully normalized gray matter values in adolescents ${ }^{20,21}$, indicates that the time-lag between admission and TP1 does not suffice to explain the lower recovery rate in adults.

Cross-sectional comparisons at TP3 demonstrated remaining decreases of cortical thickness in the superior frontal region. The residual gray matter reduction is in line with another study investigating weight restored adult AN patients, which reported persisting global reductions after reaching $90 \%$ of the ideal body weight ${ }^{23}$. However, the authors of the study did not separately analyze the influence of patients' age. In long-term recovered adult AN patients, age has not been found to be associated with gray matter measures ${ }^{10,48}$. Together with the considerable influence of age on brain restoration only in the latter part of treatment, this could indicate that brain regeneration in relatively older patients is slower, rather than incomplete, compared to younger patients.

Similar to the trajectory of cortical regeneration, the restoration of subcortical volumes showed the largest effects during the early phase of treatment with substantial increases of the amygdala, caudate nucleus, hippocampus, and thalamus. No significant increases were observed in the nucleus accumbens, pallidum, and putamen. This finding is in partial accordance with previous reports of regenerated subcortical volumes in adolescent AN patients, except for the pallidum ${ }^{20}$. The lacking regeneration of the nucleus accumbens and the putamen in our sample might be explained by the difference in age between the samples and speaks to the hypothesis of a slower restoration in adult patients. The missing correlation between age and subcortical volumes seems to challenge this interpretation. However, lower scan-rescan reliability of these structures ${ }^{49}$ may obscure a potential influence. Long-term weight-recovered AN patients have been reported to show no differences in subcortical 

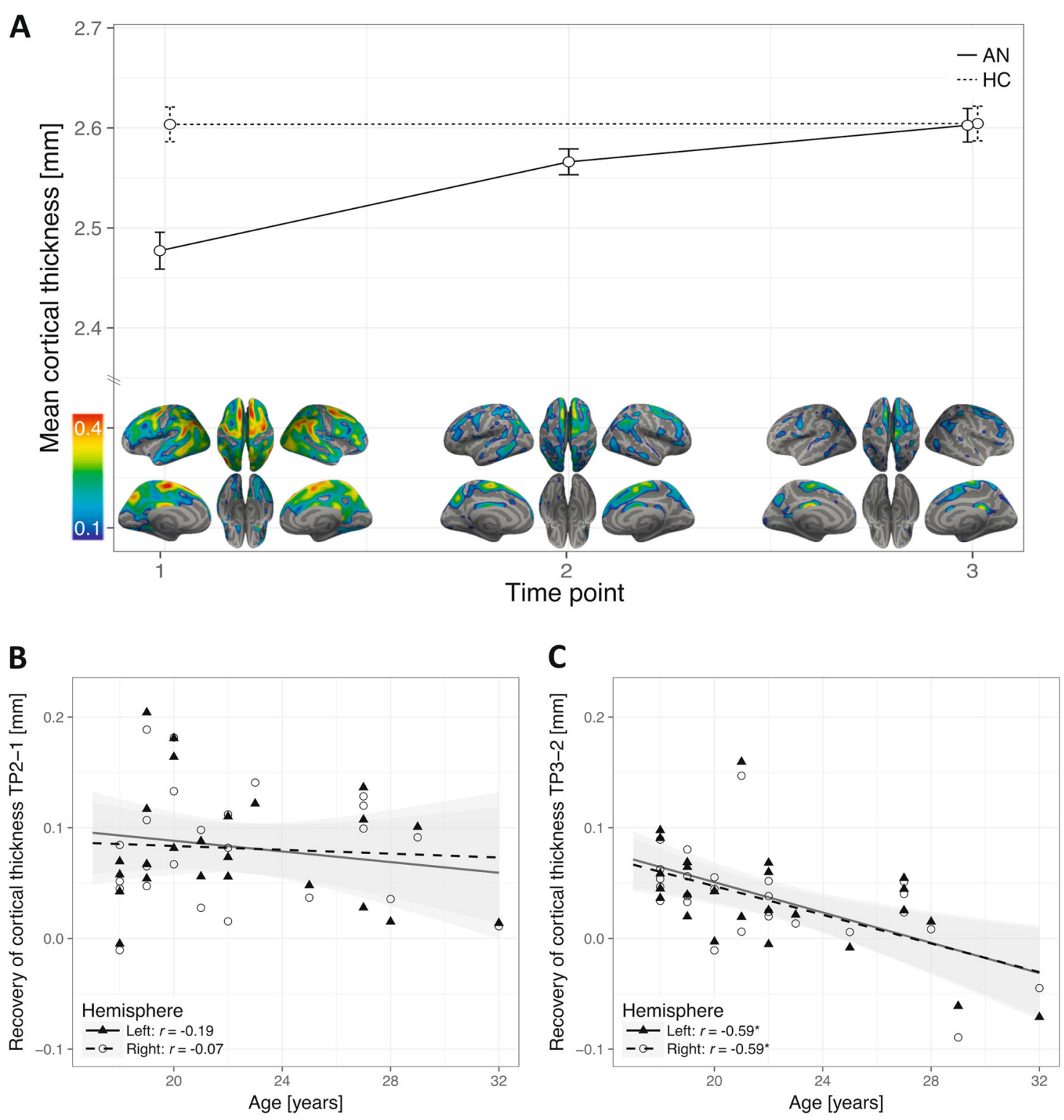

Fig. 2 Recovery of cortical thickness and correlation with patients' age. a, top: Global cortical thickness (mm) per group, left hemisphere displayed (similar trajectory for right hemisphere, not displayed). Significant increase within AN group during first (TP2-TP1: mean increase left $=$ $0.08 \mathrm{~mm} \pm 0.05, \mathrm{t}[23]=7.7, p<0.001$; mean increase right $=0.08 \mathrm{~mm} \pm 0.05, \mathrm{t}[23]=7.7, p<0.001$ ) and second (TP3-TP2: mean increase left $=$ $0.04 \mathrm{~mm} \pm 0.05, \mathrm{t}[25]=4.0, p<0.001$; mean increase right $=0.03 \mathrm{~mm} \pm 0.04, \mathrm{t}[25]=3.9, p<0.001)$ treatment phase. Larger increase during first phase than during second phase (left: mean difference of increase $=0.045 \mathrm{~mm} \pm 0.078, \mathrm{t}[23]=2.81, p<0.001$; right: mean difference of increase $=$ $0.048 \mathrm{~mm} \pm 0.077, \mathrm{t}[23]=3.06, p<0.001)$. Error bars represent $95 \%$ within-subject confidence intervals ${ }^{68} . \mathbf{a}$, bottom: Local differences in cortical thickness between groups (HC-AN) per time point, projected onto inflated brain surfaces (gray). HC values were averaged over time before differences between groups were calculated. $\mathbf{b}$ No significant correlation between age and changes in cortical thickness of AN patients between TP1 and TP2. c Significant correlations between age and recovery of cortical thickness in AN patients between TP2 and TP3; left hemisphere $R^{2}=0.35$, right hemisphere $R^{2}=0.35 .{ }^{*} p<0.05$, Holm-Bonferroni corrected.

volumes ${ }^{50}$, suggesting that recovery of these regions may occur at a later stage.

Several biological mechanisms have been discussed as explanations for the observed gray matter reduction and subsequent regeneration in AN patients, such as dehydration $^{7,17,51}$ or apoptosis (programmed cell death) of neurons or glia cells ${ }^{52,53}$. Recent reports on animal models of activity-based anorexia strongly support the hypothesis of glia cells playing a key role in $\mathrm{AN}^{54,55}$, suggesting that severe structural alterations in AN may be selectively linked to processes of astroglia and reduced glia cell proliferation $^{56}$. 
While the number of glia cells seems to stay relatively stable during healthy ageing ${ }^{57}$, the notion of diminished astrocyte proliferation ${ }^{56}$ offers an interesting explanation for the slower regeneration of cortical thickness in the older AN patients of the present study. Particularly the senescence of astrocytic cells, a protective response that permanently prevents cell proliferation ${ }^{58,59}$, increases with age $^{60}$. The correlation between patients' age and the reduced recovery of cortical thickness might thus be induced by ageing processes in glia cells, in particular diminished astrocyte proliferation. Younger AN patients would therefore have a greater capacity for full cortical restoration, which is in line with research on prognostic factors in $\mathrm{AN}$, reporting better outcomes in younger patients ${ }^{61}$. In this respect, age may be a risk factor for persisting brain impairments and a chronic illness trajectory.

\section{Clinical implications}

The findings of the present study provide strong evidence for weight restoration as an effective intervention to amend decreases of cortical and subcortical brain structures even in patients with severe AN. The strong correlation of age and regeneration of cortical thickness in the later treatment phase may indicate that younger patients have a better chance of complete cortical restitution. Age-dependent decreases in brain plasticity potentially aggravate the detrimental effects of malnutrition on the brain. This has two important clinical implications: (1) weight-restoration treatment should be started as early as possible to achieve better brain regeneration; and (2) a phase of continued guided stabilization of a healthy BMI $\left(>18.5 \mathrm{~kg} / \mathrm{m}^{2}\right)$ after weight-restoration seems important to give patients time to sufficiently recover on both a psychological and a neural level. In fact, longer weight maintenance before discharge has previously been found to be predictive of a longer time period before rehospitalization ${ }^{62}$.

\section{Limitations}

The strict weight criteria of the current study posed a challenge for patient recruitment and thus resulted in a moderate sample size. Nevertheless, our study sample is the largest to date investigating changes over three time points and the results are in line with previous structural studies with adult AN patients ${ }^{3}$. Furthermore, the longitudinal measurement of both AN patients and wellmatched $\mathrm{HC}$ allowed us to account for potential normal age-related decreases of brain structures in $\mathrm{HC}^{63-65}$ and contributes to the reliability of the findings.

The age range of the current sample was 18-32 years. While this narrow age range offers the advantage of a homogeneous group, it limits generalizability. Further longitudinal studies, including a wider age range and a follow-up after weight stabilization, are needed to clarify the long-term influence of age in this context and to elucidate mediating factors of a successful recovery of AN patients.

\section{Conclusion}

This systematic analysis of temporal patterns of structural brain recovery in adult AN patients suggests largescale reversibility of reductions in cortical thickness and subcortical volumes, with the most pronounced changes occurring in the early stages of weight-restoration treatment. Patients' age seems to have a notable influence on brain restitution, possibly reflecting decreases of brain plasticity as patients grow older.

\section{Acknowledgements}

We thank all patients and healthy controls for their contribution to this study and all members of the research team for their support, notably A. Koller, $\mathrm{S}$. Sele, C. Sob, V. Vincenti, and M. Wittlin for their help with data acquisition. Further, we gratefully acknowledge the financial support of this work from the Swiss Anorexia Nervosa Foundation (project no. 19-12), the Hermann Klaus Foundation, the EMDO Foundation, the Helene Bieber-Fonds, the Gottfried and Julia Bangerter-Rhyner-Foundation, and the Stiftung zur Förderung von Psychiatrie und Psychotherapie, Zurich, Switzerland.

\section{Author details}

${ }^{1}$ Department of Consultation-Liaison Psychiatry and Psychosomatics, University Hospital Zurich, University of Zurich, Zurich, Switzerland. ' ${ }^{2}$ ivision of Neuropsychology, Department of Psychology, University of Zurich, Zurich, Switzerland. ${ }^{3}$ Unit of Clinical and Health Psychology, Department of Psychology, University of Fribourg, Fribourg, Switzerland. ${ }^{4}$ International Normal Aging and Plasticity Imaging Center (INAPIC), University of Zurich, Zurich, Switzerland. ${ }^{5}$ University Research Priority Program (URPP) "Dynamic of Healthy Aging", University of Zurich, Zurich, Switzerland. 'Department of

Neuroradiology, University Hospital Zurich, Zurich, Switzerland. ${ }^{7}$ University of Zurich, Zurich, Switzerland

Conflict of interest

The authors declare that they have no conflict of interest.

\section{Publisher's note}

Springer Nature remains neutral with regard to jurisdictional claims in published maps and institutional affiliations.

Supplementary Information accompanies this paper at (https://doi.org/ 10.1038/s41398-020-0809-7).

Received: 8 December 2019 Revised: 15 April 2020 Accepted: 17 April 2020 Published online: 04 May 2020

\section{References}

1. American Psychiatric Association. Diagnostic and Statistical Manual of Mental Disorders (DSM-5 ${ }^{\circledR}$ ) (American Psychiatric Publishing, Inc, 2013).

2. Titova, O. E., Hjorth, O. C., Schiöth, H. B. \& Brooks, S. J. Anorexia nervosa is linked to reduced brain structure in reward and somatosensory regions: a metaanalysis of VBM studies. BMC Psychiatry 13, 110 (2013).

3. Seitz, J., Herpertz-Dahlmann, B. \& Konrad, K. Brain morphological changes in adolescent and adult patients with anorexia nervosa. J. Neural Transm. 123, 949-959 (2016).

4. Van den Eynde, F. et al. Structural magnetic resonance imaging in eating disorders: a systematic review of voxel-based morphometry studies. Eur. Eat. Disord. Rev. 20, 94-105 (2012). 
5. Phillipou, A., Rossell, S. L. \& Castle, D. J. The neurobiology of anorexia nervosa: a systematic review. Aust. N. Z. J. Psychiatry 48, 128-152 (2014).

6. Seitz, J. et al. Brain volume reduction predicts weight development in adolescent patients with anorexia nervosa. J. Psychiatr. Res. 68, 228-237 (2015).

7. Via, E. et al. Disruption of brain white matter microstructure in women with anorexia nervosa. J. Psychiatry Neurosci. 39, 367-375 (2014).

8. Fonville, L., Giampietro, V., Williams, S. C. R., Simmons, A. \& Tchanturia, K. Alterations in brain structure in adults with anorexia nervosa and the impact of illness duration. Psychol. Med. 44, 1965-1975 (2014).

9. Phillipou, A. et al. Differences in regional grey matter volumes in currently ill patients with anorexia nervosa. Eur. J. Neurosci. 47, 177-183 (2018).

10. Wagner, A. et al. Normal brain tissue volumes after long-term recovery in anorexia and bulimia nervosa. Biol. Psychiatry 59, 291-293 (2006).

11. Joos, A. et al. Grey matter deficit in long-term recovered anorexia nervosa patients. Eur. Eat. Disord. Rev. 19, 59-63 (2011).

12. Favaro, A., Tenconi, E., Degortes, D., Manara, R. \& Santonastaso, P. Gyrification brain abnormalities as predictors of outcome in anorexia nervosa. Hum. Brain Mapp. 36, 5113-5122 (2015).

13. Bang, L., Rø, Ø. \& Endestad, T. Normal gray matter volumes in women recovered from anorexia nervosa: a voxel-based morphometry study. BMC Psychiatry 16, 144 (2016).

14. King, J. A. et al. Global cortical thinning in acute anorexia nervosa normalizes following long-term weight restoration. Biol. Psychiatry 77, 624-632 (2015).

15. Nickel, K. et al. Recovery of cortical volume and thickness after remission from acute anorexia nervosa. Int. J. Eat. Disord. 51, 1056-1069 (2018).

16. Friederich, H.-C. et al. Grey matter abnormalities within cortico-limbic-striatal circuits in acute and weight-restored anorexia nervosa patients. Neuroimage 59, 1106-1113 (2012).

17. Frank, G. K. W., Shott, M. E., Hagman, J. O. \& Mittal, V. A. Alterations in brain structures related to taste reward circuitry in ill and recovered anorexia nervosa and in bulimia nervosa. Am. J. Psychiatry 170, 1152-1160 (2013).

18. Lavagnino, L. et al. Cortical thickness patterns as state biomarker of anorexia nervosa. Int. J. Eat. Disord. 51, 241-249 (2018).

19. Castro-Fornieles, J. et al. Cortical thickness 20 years after diagnosis of anorexia nervosa during adolescence. Eur. Arch. Psychiatry Clin. Neurosci. https://doi.org/ 10.1007/s00406-019-00992-4 (2019).

20. Bernardoni, F. et al. Weight restoration therapy rapidly reverses cortical thinning in anorexia nervosa: a longitudinal study. Neuroimage 130, 214-222 (2016).

21. Bomba, M. et al. Global and regional brain volumes normalization in weightrecovered adolescents with anorexia nervosa: preliminary findings of a longitudinal voxel-based morphometry study. Neuropsychiatr. Dis. Treat. 11, 637 (2015).

22. Mainz, V., Schulte-Rüther, M., Fink, G. R., Herpertz-Dahlmann, B. \& Konrad, K. Structural brain abnormalities in adolescent anorexia nervosa before and after weight recovery and associated hormonal changes. Psychosom. Med. 74 574-582 (2012).

23. Roberto, C. A. et al. Brain tissue volume changes following weight gain in adults with anorexia nervosa. Int. J. Eat. Disord. 44, 406-411 (2011).

24. Swayze, V. W. et al. Brain tissue volume segmentation in patients with anorexia nervosa before and after weight normalization. Int. J. Eat. Disord. 33, 33-44 (2003).

25. First, M. B., Spitzer, R. L., Gibbon, M. \& Williams, J. B. W. Structured Clinical Interview for DSM-IV-TR Axis I Disorders, Research Version, Patient Edition (SCID-I) P) (New York State Psychiatric Institute, New York, NY, 2002).

26. Oldfield, R. C. The assessment and analysis of handedness: the Edinburgh inventory. Neuropsychologia 9, 97-113 (1971).

27. Kaufmann, L.-K. et al. Fornix under water? Ventricular enlargement biases forniceal diffusion magnetic resonance imaging indices in anorexia nervosa. Biol. Psychiatry Cogn. Neurosci. Neuroimaging 2, 430-437 (2017).

28. Frank, G. K. W., Favaro, A., Marsh, R., Ehrlich, S. \& Lawson, E. A. Toward valid and reliable brain imaging results in eating disorders. Int. J. Eat. Disord. 51, 250-261 (2018).

29. King, J. A., Frank, G. K. W., Thompson, P. M. \& Ehrlich, S. Structural neuroimaging of anorexia nervosa: future directions in the quest for mechanisms underlying dynamic alterations. Biol. Psychiatry 83, 224-234 (2018).

30. Formann, A. K. \& Piswanger, K. Wiener Matrizen-Test. Manual (Beltz Test Gesellschaft, Weinheim, 1979).

31. Schmidt, K.-H. \& Metzler, P. Wortschatztest (WST) (Beltz Test GmbH, Weinheim, 1992).
32. Hänsgen, K.-D. Hogrefe TestSystem (Systemhandbuch. Hogrefe, Göttingen, 2006).

33. Hilbert, A. \& Tuschen-Caffier, B. Eating Disorder Examination-Questionnaire (Verlag für Psychotherapie, PAG Institut für Psychologie AG, Münster, 2006).

34. Hautzinger, M., Bailer, M., Worall, H. \& Keller, F. Beck-Depressions-Inventar (BDI). Bearbeitung der deutschen Ausgabe (Verlag Hans Huber, Bern, 1994).

35. Dale, A. M., Fischl, B. \& Sereno, M. I. Cortical surface-based analysis. I. Segmentation and surface reconstruction. Neuroimage 9, 179-194 (1999).

36. Reuter, M., Schmansky, N. J., Rosas, H. D. \& Fischl, B. Within-subject template estimation for unbiased longitudinal image analysis. Neuroimage $\mathbf{6 1}$, 1402-1418 (2012).

37. Reuter, M. \& Fischl, B. Avoiding asymmetry-induced bias in longitudinal image processing. Neuroimage 57, 19-21 (2011).

38. Reuter, M., Rosas, H. D. \& Fischl, B. Highly accurate inverse consistent registration: a robust approach. Neuroimage 53, 1181-1196 (2010).

39. Fischl, B. et al. Whole brain segmentation: automated labeling of neuroanatomical structures in the human brain. Neuron 33, 341-355 (2002).

40. Fischl, B. et al. Automatically parcellating the human cerebral cortex. Cereb. Cortex 14, 11-22 (2004).

41. Bernal-Rusiel, J. L., Greve, D. N., Reuter, M., Fischl, B. \& Sabuncu, M. R. Statistical analysis of longitudinal neuroimage data with Linear Mixed Effects models. Neuroimage 66, 249-260 (2013).

42. Bernal-Rusiel, J. L., Reuter, M., Greve, D. N., Fischl, B. \& Sabuncu, M. R. Spatiotemporal linear mixed effects modeling for the mass-univariate analysis of longitudinal neuroimage data. Neuroimage 81, 358-370 (2013).

43. Benjamini, Y., Krieger, A. M. \& Yekutieli, D. Adaptive linear step-up procedures that control the false discovery rate. Biometrika 93, 491-507 (2006).

44. Holm, S. A simple sequentially rejective multiple test procedure. Scand. J. Stat. 6, 65-70 (1979).

45. R Core Team. R: a language and environment for statistical computing https:// www.r-project.org/ (2018).

46. Hedges, L. V. Distribution theory for Glass's estimator of effect size and related estimators. J. Educ. Stat. 6, 107 (1981).

47. Pascual-Leone, A. et al. Characterizing brain cortical plasticity and network dynamics across the age-span in health and disease with TMS-EEG and TMSfMRI. Brain Topogr. 24, 302-315 (2011).

48. Mühlau, M. et al. Gray matter decrease of the anterior cingulate cortex in anorexia nervosa. Am. J. Psychiatry 164, 1850-1857 (2007).

49. Guadalupe, T. et al. Measurement and genetics of human subcortical and hippocampal asymmetries in large datasets. Hum. Brain Mapp. 35, 3277-3289 (2014).

50. Miles, A. E., Voineskos, A. N., French, L. \& Kaplan, A. S. Subcortical volume and cortical surface architecture in women with acute and remitted anorexia nervosa: an exploratory neuroimaging study. J. Psychiatr. Res. 102, 179-185 (2018).

51. Frank, G. K. W. What causes eating disorders, and what do they cause? Biol. Psychiatry 77, 602-603 (2015).

52. Ehrlich, S. et al. Glial and neuronal damage markers in patients with anorexia nervosa. J. Neural Transm. 115, 921-927 (2008).

53. Ehrlich, S. et al. S100B in underweight and weight-recovered patients with anorexia nervosa. Psychoneuroendocrinology 33, 782-788 (2008).

54. Frintrop, L. et al. The reduction of astrocytes and brain volume loss in anorexia nervosa - the impact of starvation and refeeding in a rodent model. Transl. Psychiatry 9, 159 (2019).

55. Frintrop, L. et al. Reduced astrocyte density underlying brain volume reduction in activity-based anorexia rats. World J. Biol. Psychiatry 19, 225-235 (2018).

56. Barbarich-Marsteller, N. C. et al. Activity-based anorexia is associated with reduced hippocampal cell proliferation in adolescent female rats. Behav. Brain Res. 236, 251-257 (2013).

57. Weissleder, C. et al. Decline in proliferation and immature neuron markers in the human subependymal zone during aging: relationship to EGF- and FGFrelated transcripts. Front. Aging Neurosci. 8, 1-12. (2016).

58. Campisi, J. Cellular senescence as a tumor-suppressor mechanism. Trends Cell Biol. 11, S27-S31. (2001)

59. Collado, M. \& Serrano, M. Senescence in tumours: evidence from mice and humans. Nat. Rev. Cancer 10, 51-57 (2010).

60. Chinta, S. J. et al. Cellular senescence and the aging brain. Exp. Gerontol. 68, 3-7 (2015). 
61. Le Grange, D. et al. Predictors and moderators of outcome for severe and enduring anorexia nervosa. Behav. Res. Ther. 56, 91-98 (2014).

62. Lay, B., Jennen-Steinmetz, C., Reinhard, I. \& Schmidt, M. H. Characteristics of inpatient weight gain in adolescent anorexia nervosa: relation to speed of relapse and re-admission. Eur. Eat. Disord. Rev. 10, 22-40 (2002).

63. Tamnes, C. K. et al. Brain maturation in adolescence and young adulthood: regional age-related changes in cortical thickness and white matter volume and microstructure. Cereb. Cortex 20, 534-548 (2010).

64. Salat, D. H. et al. Thinning of the cerebral cortex in aging. Cereb. Cortex $\mathbf{1 4}$ 721-730 (2004).
65. Lemaitre, $H$. et al. Normal age-related brain morphometric changes: Nonuniformity across cortical thickness, surface area and gray matter volume? Neurobiol. Aging 33, 617.e1-617.e9 (2012).

66. Riedel, $M$. et al. Response and remission criteria in major depression-a validation of current practice. J. Psychiatr. Res. 44, 1063-1068 (2010).

67. $R \varnothing, \varnothing$., Reas, D. L. \& Stedal, K. Eating disorder examination questionnaire (EDEQ) in Norwegian adults: discrimination between female controls and eating disorder patients. Eur. Eat. Disord. Rev. 23, 408-412 (2015).

68. Morey, R. D. Confidence intervals from normalized data: a correction to Cousineau (2005). Tutor. Quant. Methods Psychol. 4, 61-64 (2008). 